\title{
Similarity Law on Shedding Frequency of Cavitation Cloud Induced by a Cavitating Jet*
}

\author{
Satoshi NISHIMURA**, Osamu TAKAKUWA** and Hitoshi SOYAMA** \\ ${ }^{* *}$ Department of Nanomechanics, Graduate School of Engineering, Tohoku University, \\ 6-6-01 Aoba, Aramaki, Aoba-ku, Sendai, Miyagi 980-8579, Japan \\ E-mail: o_takakuwa@mm.mech.tohoku.ac.jp
}

\begin{abstract}
In order to clarify the mechanism of cavitation cloud shedding, the shedding frequencies of cavitation clouds were investigated by high-speed observations and image analysis. The results indicate that the shedding frequency follows the similarity law for unsteady vortex flow. The ranges of parameters considered were from 0.4 to $2.0 \mathrm{~mm}$ for the nozzle throat diameter, from 10 to $30 \mathrm{MPa}$ for the injection pressure, and from 0.01 to 0.05 for the cavitation number. The similarity law is discussed with reference to the Strouhal number, which depends on the shedding frequency of the cavitation cloud, the width of the cavitating jet and the jet velocity at the nozzle exit. Formulas describing the relationships between the shedding frequency of the cavitation cloud and the investigated parameters were developed. In addition, the Strouhal number for cavitation cloud shedding was shown to be independent of the investigated parameters and constant, with a value of 0.18 . In fact, the cavitation cloud shedding was governed by the constant Strouhal number.
\end{abstract}

Key words: Cavitation, Jet, Nozzle, Image Analysis, Frequency

\section{Introduction}

Cavitating jets, i.e., submerged high-speed water jets, have been successfully applied in industry for, for example, metallic material peening ${ }^{(1)}$, underwater rock cutting ${ }^{(2)}$ and aiding chemical reactions ${ }^{(3)}$. As is well known, a cavitating jet exhibits periodic shedding of the cavitation cloud, the frequency of which is closely related to the jet performance. Thus, to facilitate the practical use of a cavitating jet, the mechanism and the parameters that affect cavitation cloud shedding should be investigated. In this study, in order to clarify the mechanism of cavitation cloud shedding, high-speed video observations were made and image analyses were done, which showed the shedding frequency of the cavitation cloud to be governed by a similarity law.

When pressurized water is injected into a water filled chamber through a nozzle, small cavitation vortices are generated in areas of low pressure in the shear layer, near the nozzle exit ${ }^{(4-7)}$. The cavitation vortices merge with one another, forming large discrete cavitating structures, i.e., cavitation clouds ${ }^{(8)}$. Past studies have shown that cavitation clouds are discharged and shed periodically, which introduces a periodic collapse of the cavitation cloud $^{(9-11)}$. As a cavitating jet utilizes the energy released on impact as the cavitation cloud collapses, it is obvious that the shedding frequency is an important factor affecting the aggressive intensity of the cavitating jet. However, most of the significant studies concerning cavitating jet characteristics focus on cavitation inception ${ }^{(4-7)}$, which is also an important aspect affecting the jet performance. Hence, little is known about the shedding behavior of the cavitation cloud.

Received 3 Sep., 2012 (No. 12-0353)

DOI: 10.1299/jst.7.405]

Copyright $\odot 2012$ by JSME 
Visualizing the cavitating jet is an effective method for investigating the instantaneous unsteady behavior of cavitation phenomena. The cavitation inception characteristics of a submerged water jet such as the scale, boundary thickness and flow instability effect have been revealed by holography and still photography by Ooi ${ }^{(4)}$, Ran and $\mathrm{Katz}^{(5)}$, Gopalan et al. ${ }^{(6)}$ and Straka et al. ${ }^{(7)}$. These studies have contributed significantly to the understanding of cavitation inception in a jet flow; however, little attention was paid to the cavitation behavior after the inception process. Pioneering observations focusing on the behavior of large scale cavitating structures produced when cavitation bubbles merge were conducted by Chahine et al, by high-speed observations of deep hole drilling with a cavitating jet ${ }^{(9)}$. The cavitating jet was observed to self resonate and periodically discharge a cloud like cavitating structure, which changed its shape and discharge frequency with cavitation number. Soyama et al. demonstrated that the injection pressure of the cavitating jet also affects the shedding frequency of the cavitation cloud, and suggested that the complex pressure gradient conditions around the cavitating jet induce cavitation cloud shedding ${ }^{(10)}$. Similar trends were confirmed in the study by Hulti and Nedeljkovic ${ }^{(11)}$. Sato et al. analyzed the shedding behavior of a cavitation cloud by the frame difference method, and concluded that the shedding is caused by the re-entrant motion toward the nozzle, accompanied by cavitation clouds collapsing in the downstream section ${ }^{(12)}$. However, it should be noted that none of the prior studies have attempted to formulate the relationship between the shedding frequency of the cavitation cloud and the parameters affecting it.

Therefore in this study, the main effort was addressed to formulating the relationships between the cavitation cloud shedding frequency and the various parameters that affect it, with reference, in particular, to the similarity law for jet flow. The effect of the nozzle throat diameter, injection pressure and cavitation number on the shedding frequency of the cavitation cloud induced by the cavitating jet was investigated by high-speed observation and image analysis. Subsequently, experimental formulas giving the relationships between the shedding frequency and the parameters were obtained. Finally, the Strouhal number of cavitation cloud shedding, which depends on the shedding frequency of the cavitation cloud, the width of the cavitating jet and the jet velocity at the nozzle exit, was investigated. The mechanism and the similarity law for cavitation cloud shedding are also discussed.

\section{Experimental Facilities and Procedures}

A schematic diagram of the cavitating jet apparatus used for the experiments is shown in Fig. 1. The high-speed water jet is pressurized by a plunger pump to a maximum pressure of $35 \mathrm{MPa}$ and has a maximum discharge of $3.0 \times 10^{-2} \mathrm{~m}^{3} / \mathrm{min}$, and is injected into the test section through the nozzle. The test section is a cylindrical vessel of $285 \mathrm{~mm}$ diameter and $240 \mathrm{~mm}$ length. The nozzle is placed on the wall of the vessel, as Fig. 1 shows. The injection pressure, $p_{1}$, is controlled by the speed of the inverter motor connected to the plunger pump. The test section's ambient pressure, $p_{2}$, is controlled by the downstream valve.

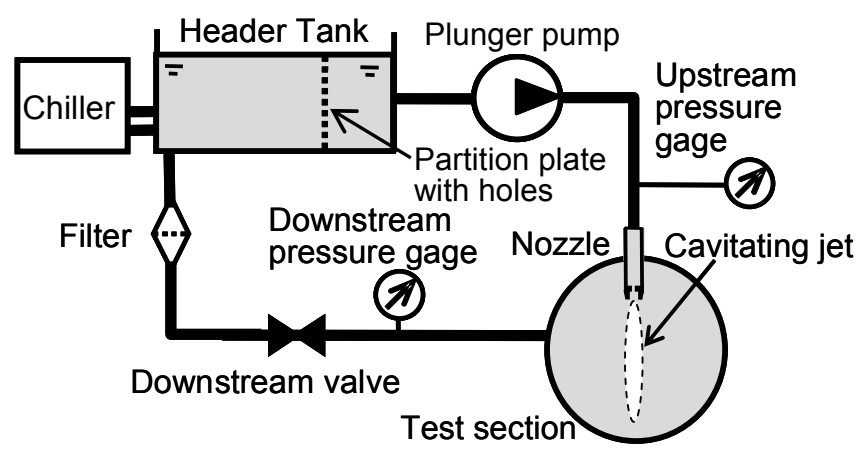

Fig. 1 Cavitating jet apparatus 
The test water is recirculated into the header tank, and residual bubbles are removed by the partition plate with holes. Pressure transducers are placed on both the upstream and downstream sections of the nozzle in order to measure the injection pressure and ambient pressure, respectively. The water temperature is controlled by a chiller, and kept constant at $300 \pm 3 \mathrm{~K}$.

The main parameter of a cavitating jet is the cavitation number, $\sigma^{(13)}$. Generally, the cavitation number is defined by the ratio of the hydrodynamic pressure to the static pressure in the cavitating flow ${ }^{(14)}$. In the case of a nozzle or orifice, the hydrodynamic pressure can be expressed by the pressure difference between the upstream and downstream sections of the nozzle or orifice. Thus, the cavitation number of a cavitating jet is given by Eq. (1),

$$
\sigma=\frac{p_{2}-p_{v}}{p_{1}-p_{2}} \cong \frac{p_{2}}{p_{1}}
$$

where $p_{1}$ is the injection pressure, $p_{2}$ the ambient pressure, and $p_{v}$ the vapor pressure of the test water. The cavitation number can be simplified, as in Eq. (1), since in the present case $p_{1} \gg p_{2} » p_{v}$.

The cavitating jet nozzle is shown in Fig. 2. The nozzle throat has a cylindrical shape, with variable diameter, $d$, which in our experiments was $0.4,0.6,1.0,1.5$ and $2.0 \mathrm{~mm}$. The bore, $D$, and length, $L$, at the outlet of the nozzle were set such that the ratio $d: D: L$ was equal to 1: $8: 8$, since this affects the shedding frequency of the cavitation cloud, and the aggressive intensity of the jet is a maximum with this geometry ${ }^{(15)}$. The thickness of the nozzle plate was kept constant at $3 d$.

The cavitating conditions used in this study are shown in Table 1. The injection pressure, $p_{1}$, was varied in the range from 10 to $30 \mathrm{MPa}$. The range of the cavitation number was from 0.01 to 0.05 , which was varied by changing $p_{1}$ and the ambient pressure, $p_{2}$. However, in order to investigate the effect of $p_{1}$ only, $\sigma$ should be kept constant. $p_{\sigma}$ represents the ambient pressure at which the cavitation number, $\sigma$, is driven to 0.014 at each injection pressure. It is known that the maximum aggressive intensity of the cavitating jet occurs at $\sigma=0.014^{(16)}$, and therefore this value was chosen. High-speed observations were conducted for each nozzle throat diameter and cavitating condition.

The cavitating jet was recorded with a high-speed video camera (Redlake Motion Pro HS-4) through an acrylic window in the test section. Two halogen lights and one xenon lamp were placed on the same side as the video camera, as the jet appears white in the image.

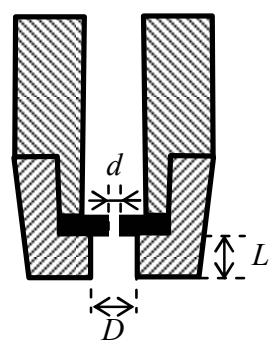

Fig. 2 Nozzle outlet geometry

Table 1 Cavitation test conditions

\begin{tabular}{cccccc}
\hline \hline Ambient & \multicolumn{5}{c}{ Injection pressure $p_{1} \mathrm{MPa}$} \\
\cline { 2 - 6 } pressure $p_{2} \mathrm{MPa}$ & 10 & 15 & 20 & 25 & 30 \\
\hline 0.2 & 0.02 & 0.013 & 0.01 & - & - \\
0.3 & 0.03 & 0.02 & 0.015 & 0.012 & 0.01 \\
0.4 & 0.04 & 0.027 & 0.02 & 0.016 & 0.013 \\
0.5 & 0.05 & 0.033 & 0.025 & 0.02 & 0.017 \\
$p_{\sigma}$ & 0.014 & 0.014 & 0.014 & 0.014 & 0.014 \\
\hline \hline
\end{tabular}




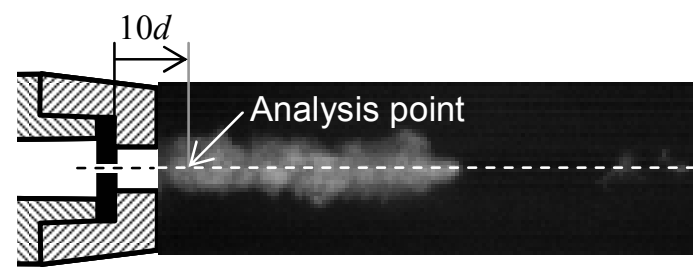

Fig. 3 Analysis point chosen as $10 d$

So as to observe the jet clearly, an exposure time of $2 \mu \mathrm{s}$, frame rate of 20,000 fps and frame size of $128 \times 512$ pixels were chosen. $1 \mathrm{~mm}$ corresponded to 1.5 pixels in the image.

The shedding frequency of the cavitation cloud was calculated from the fluctuation in the gray scale value at the analysis point in the image. As shown in Fig. 3, the analysis point was chosen as $x / d=10$ at the center of the jet ${ }^{(17)}$, where $x$ denotes the distance from the nozzle exit. The effect of the position of the analysis point on the shedding frequency of the cavitation cloud is discussed in the Appendix. As the gray scale value varies with the distribution of the cavitation cloud, the cyclic fluctuations of the grayscale value clearly indicate the discharge and shedding of the cavitation cloud. These cyclic fluctuations were transformed into a frequency spectrum using a Fourier transform program. The shedding frequency of the cavitation cloud, $f_{\text {shedd }}$, was obtained from the average frequency of the 20 largest peaks in the spectrum, and the measurement uncertainty was considered to be the standard deviation of the frequency of these peaks.

The width of the cavitating jet, $w$, was also studied. A composite picture was made by superimposing 2,000 frames taken by the high-speed video camera. The edges of the jet were determined as the points where the slope of the gray scale value to the width direction is 0 , as Fig. 4 shows. Then, the maximum width of the jet was scanned and equated to $w$.

In order to discuss the similarity law for the shedding frequency, the Strouhal number, $S t$, was calculated. The definition of the Strouhal number for cavitating flow should be carefully considered, as different definitions can lead to different results. For a cavitating jet, $S t$ is conventionally based on the nozzle throat diameter, $d$. However, to take into consideration the effect of the cavitation number on the size of the cavitation cloud, St based on the width of the cavitating jet, $w$, seems to be more suitable, as $w$ depends on the cavitation number. Thus, in the present paper, $S t$ is defined by the shedding frequency of the cavitation cloud, $f_{\text {shedd }}$, the width of the cavitating jet, $w$, and the velocity of the jet at the nozzle exit, $U$, and is given by Eq. (2)

$$
S t=\frac{f_{\text {shedd }} \cdot w}{U}
$$

The velocity of the jet at the nozzle exit, $U$, is obtained from Eq. (3)

$$
U=\sqrt{\frac{2\left(p_{1}-p_{2}\right)}{\rho}} \cong \sqrt{\frac{2 p_{1}}{\rho}}
$$

where $p_{1}$ is the injection pressure, $p_{2}$ the ambient pressure, $\rho$ the density of water, and $p_{1} \gg p_{2}$. A cavitating jet is a typical unsteady vortex flow. Taking account of the similarity law for an unsteady vortex flow ${ }^{(18)}$, it can be assumed that the Strouhal number, $S t$, for cavitation cloud

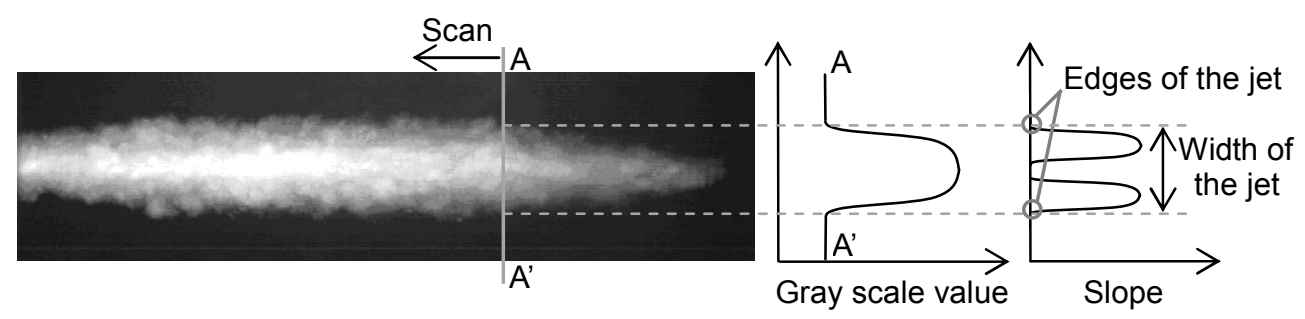

Fig. 4 Composite picture of a cavitating jet 
shedding is constant, even though the nozzle throat diameter, $d$, injection pressure, $p_{1}$, and cavitation number, $\sigma$, vary. With $S t$ constant the following proportionalities are obtained from Eqs. (2) and (3)

$$
\begin{aligned}
& f_{\text {shedd }} \propto w^{-1} \\
& f_{\text {shedd }} \propto p_{1}^{0.5}
\end{aligned}
$$

Equations (4) and (5) are compared with the experimentally derived relationships, and the validity of the equations is discussed.

\section{Results}

Figure 5 shows cavitation cloud shedding under various cavitating conditions, with $d=$ $1 \mathrm{~mm}$. The flow direction is from left to right. The white masses appearing in Fig. 5 are the cavitation clouds, which can be seen to discharge and shed periodically. As shown in Fig. 5(a), the cavitation cloud breaks 4 times around the nozzle exit at $0.5,0.9,1.4$ and $1.9 \mathrm{~ms}$ for $p_{1}=20 \mathrm{MPa}$ and $\sigma=0.014$. Therefore, the shedding frequency of the cavitation cloud can be estimated to be $2.0 \mathrm{kHz}$ under these conditions. In the same way, the shedding frequency of the cavitation cloud with $p_{1}=30 \mathrm{MPa}$ and $\sigma=0.014$ can be estimated to be $2.4 \mathrm{kHz}$ and that with $p_{1}=20 \mathrm{MPa}$ and $\sigma=0.02$ to be $3.2 \mathrm{kHz}$. Thus, this demonstrates that the shedding frequency of the cavitation cloud increases with increasing injection pressure from $20 \mathrm{MPa}$ to $30 \mathrm{MPa}$ and cavitation number from 0.014 to 0.02 . It is also shown that the width of the cavitation cloud, as well as the length decreases with increasing cavitation number from 0.014 to 0.02 .

Figure 6 shows the width of the cavitating jet normalized by the nozzle throat diameter, $w / d$, for various cavitation numbers, $\sigma$. The normalized width of the cavitating jet, is almost constant for constant $\sigma$, even though $d$ varies by a factor of 5 . Thus, the following relationship between $w$ and $d$ holds

$w \propto d$

Using this relationship, Eq. (4) can now be rewritten as follows;

$$
f_{\text {shedd }} \propto d^{-1}
$$

In Fig. $6, w$ clearly decreases with increasing $\sigma$, irrespective of the diameter, $d$. This tendency suggests that it is difficult to develop cavitation with low injection pressure and high ambient pressure conditions. The relationship between $w$ and $\sigma$ was obtained using the least squares method, and is given in Eq. (8). The standard deviation of the power index was calculated, and is considered to be the measurement error range.

$w \propto \sigma^{-0.76 \pm 0.02}$

Soyama and Lichtarowicz reported a similar value for the power index for the exponential relationship between the cavitating length and cavitation number ${ }^{(17)}$. Thus, the cavitating region is likely to vary with cavitation number in the same manner in both axial and radial directions. Finally, using Eq. (8), Eq. (4) can be rewritten as follows;

$$
f_{\text {shedd }} \propto \sigma^{0.76 \pm 0.02}
$$

Figure 7 shows the variation of the frequency spectrum with different parameters, obtained from the image analysis. It can be seen in Fig. 7 (a) that there is a spectral peak around $2.0 \mathrm{kHz}$ when the nozzle throat diameter, $d$, is $1 \mathrm{~mm}$, as observed in Fig. 5 (a). Thus, it is obvious that the peak around $2.0 \mathrm{kHz}$ corresponds to the shedding frequency of the cavitation cloud. The peak frequency shifts to lower values, as $d$ increases. For example, the peak frequency is located at around $6.0 \mathrm{kHz}$ with $d=0.4 \mathrm{~mm}$, while in the case of $d=2 \mathrm{~mm}$ 
the peak is located at around $1.0 \mathrm{kHz}$. In Fig. 7 (b), the peak frequency is found to shift slightly to higher values with increasing $p_{1}$. The peak frequency is located at around 1.8 $\mathrm{kHz}$ for $p_{1}=10 \mathrm{MPa}$ and $2.4 \mathrm{kHz}$ for $p_{1}=30 \mathrm{MPa}$, confirming the results from Fig. 5 (b). Fig. 7 (c) demonstrates that the peak frequency shifts to higher values with increasing $\sigma$. The peak frequency shifts from around $2 \mathrm{kHz}$ to $4 \mathrm{kHz}$, as $\sigma$ increases from 0.01 to 0.02 . It should be noted that the measurement uncertainty increases and the profile broadens as the peak moves to higher frequencies.

Figures 8 and 9 show the variation of shedding frequency, $f_{\text {shedd, }}$, with the nozzle throat diameter, $d$. The cavitation number is kept constant in Fig. 8, while the injection pressure remains constant in Fig. 9. It can be seen that there is a uniform trend in the figures in that $f_{\text {shedd }}$ decreases with increasing $d$, as expected from Eq. (7). The experimental
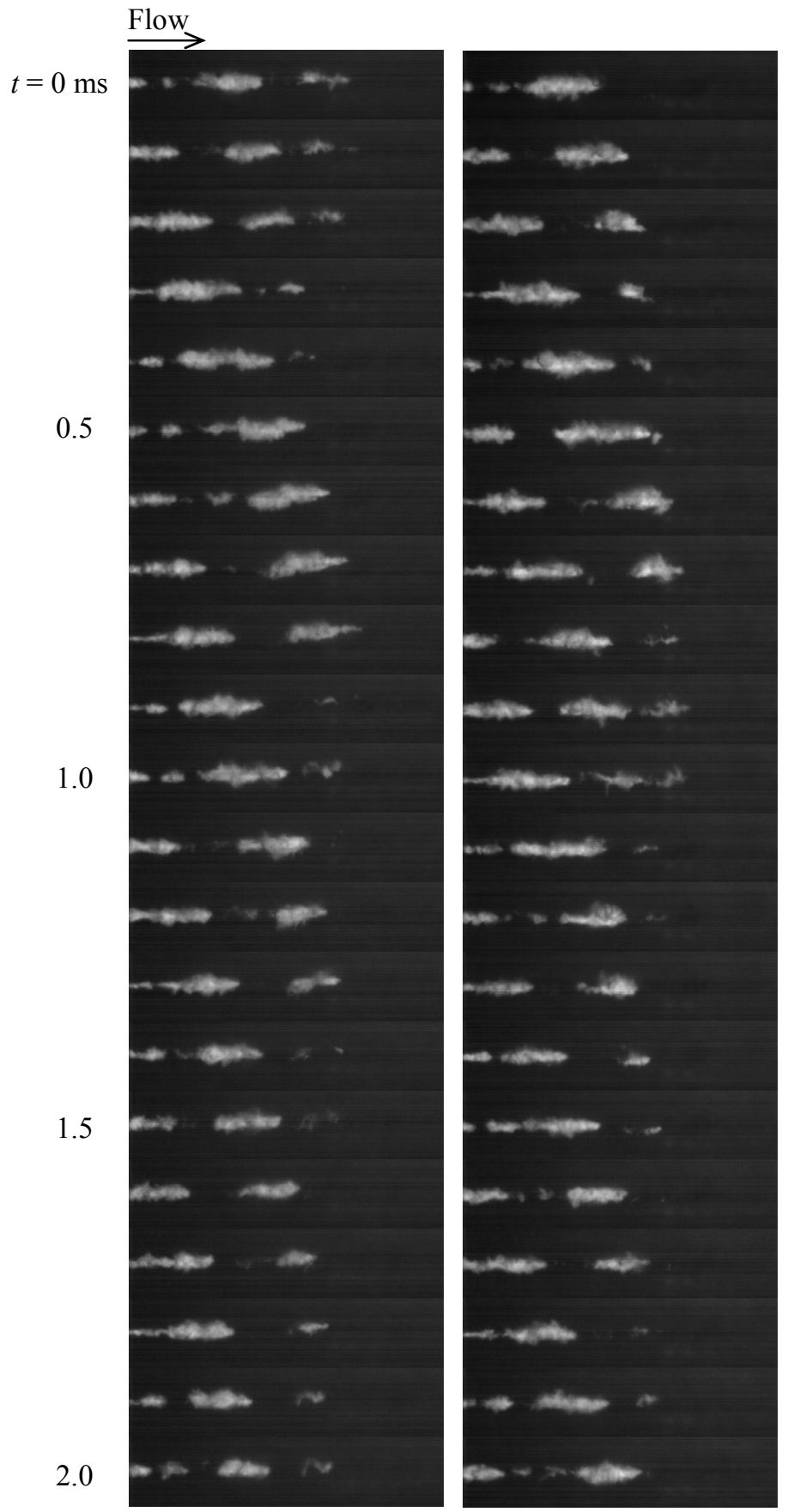

(a) $p_{1}=20 \mathrm{MPa}, \sigma=0.014$ (b) $p_{1}=30 \mathrm{MPa}, \sigma=0.014$

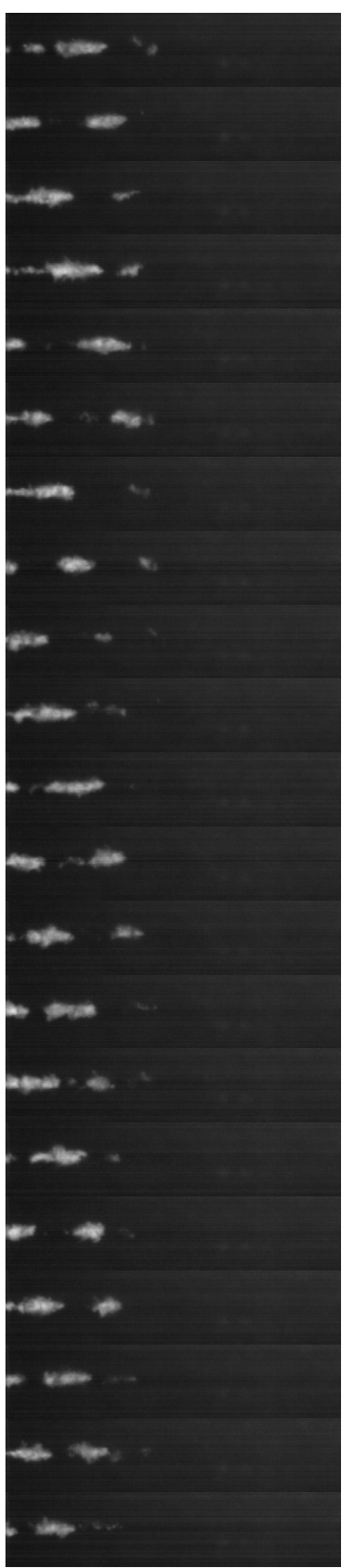

(c) $p_{1}=20 \mathrm{MPa}, \sigma=0.02$

Fig. 5 Cavitating jet under various conditions 


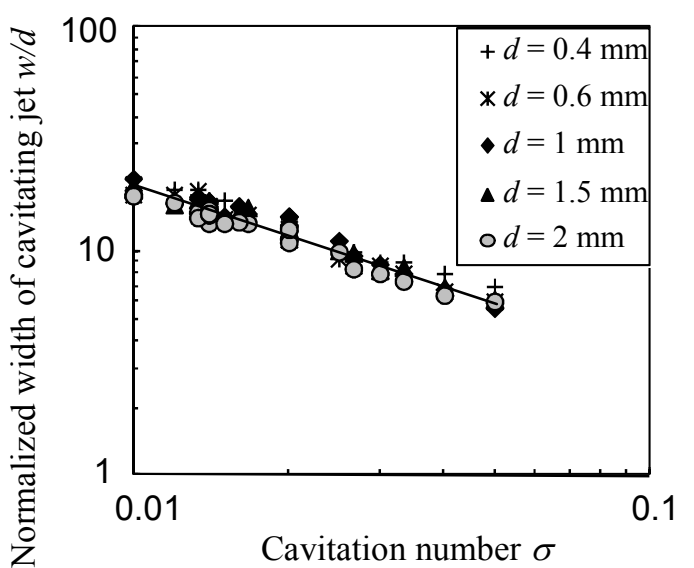

Fig. 6 Variation of normalized width of cavitating jet with cavitation number

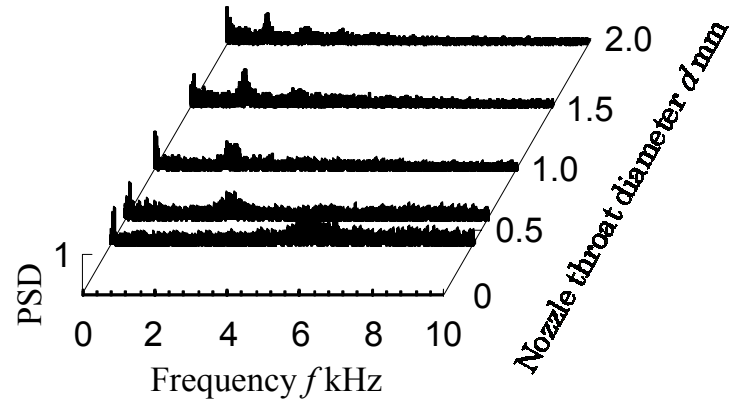

(a) Frequency spectrum varying with nozzle throat diameter $\left(p_{1}=20 \mathrm{MPa}, \sigma=0.014\right)$

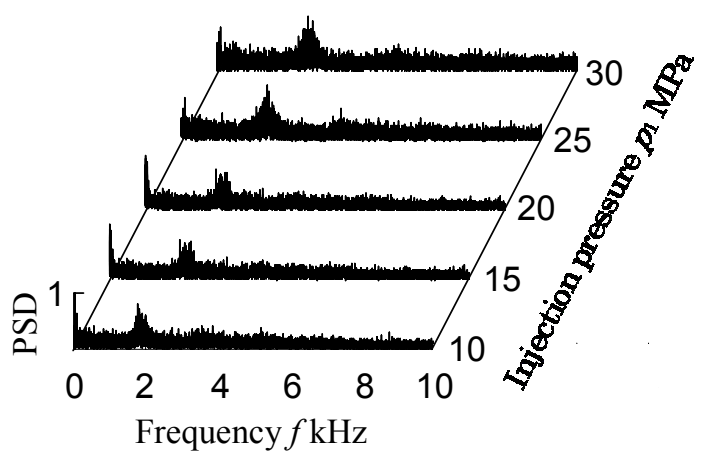

(b) Frequency spectrum varying with injection pressure $(d=1 \mathrm{~mm}, \sigma=0.014)$

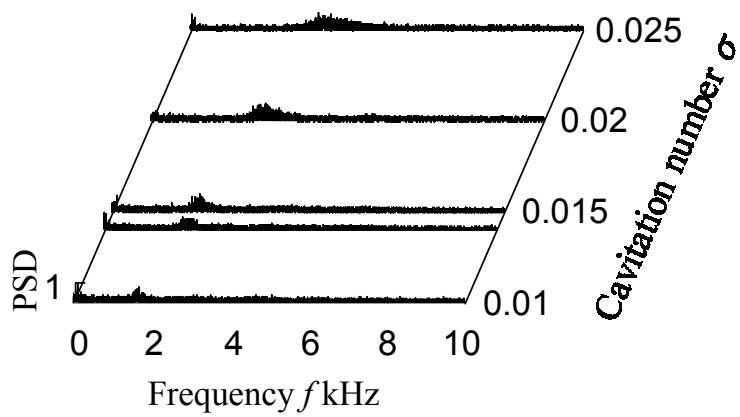

(c) Frequency spectrum varying with cavitation number $\left(d=1 \mathrm{~mm}, p_{1}=20 \mathrm{MPa}\right)$

Fig. 7 Frequency spectrum varying with different parameters 


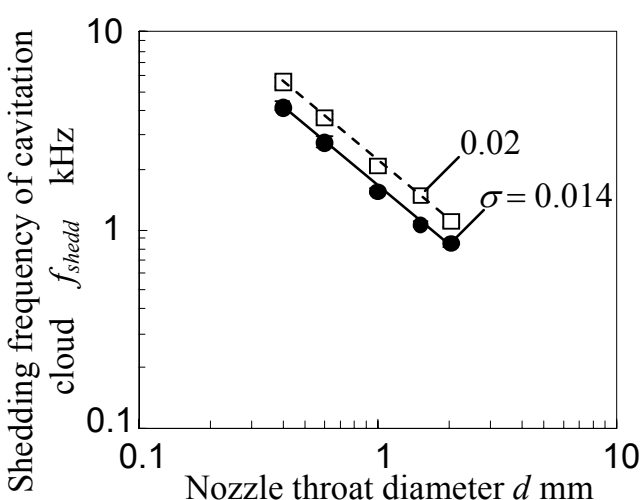

(a) $p_{1}=10 \mathrm{MPa}$

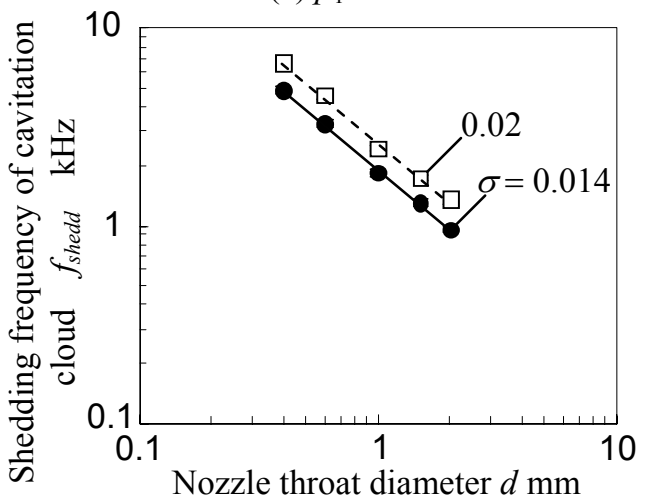

(b) $p_{1}=15 \mathrm{MPa}$

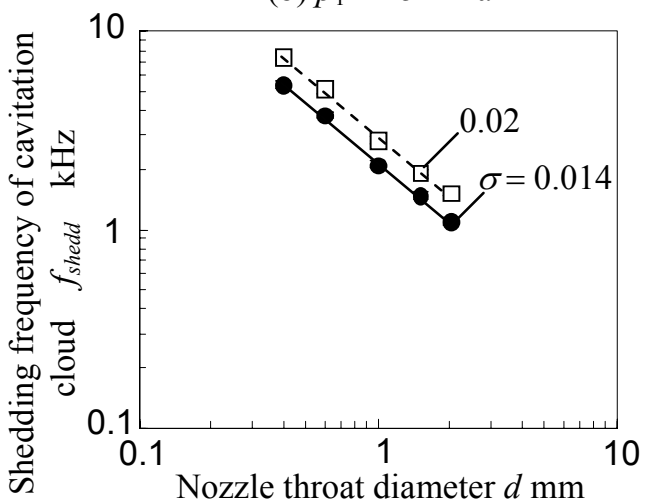

(c) $p_{1}=20 \mathrm{MPa}$

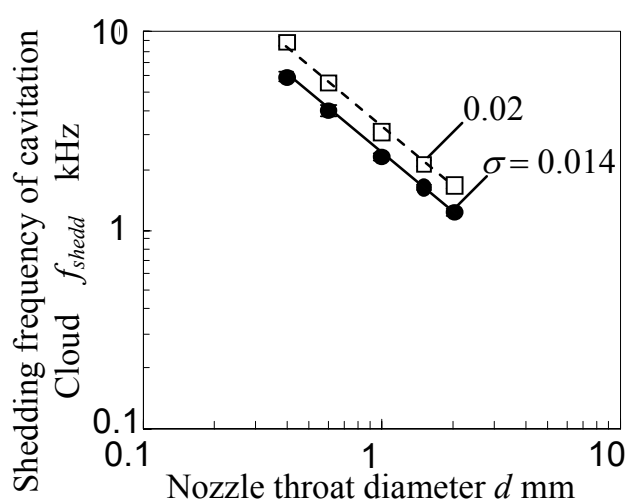

(d) $p_{1}=25 \mathrm{MPa}$

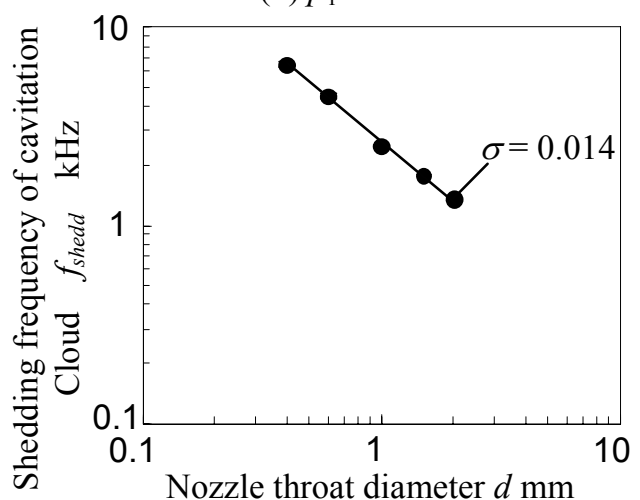

(e) $p_{1}=30 \mathrm{MPa}$

Fig. 8 Variation of the shedding frequency of a cavitation cloud with nozzle throat diameter at constant cavitation number

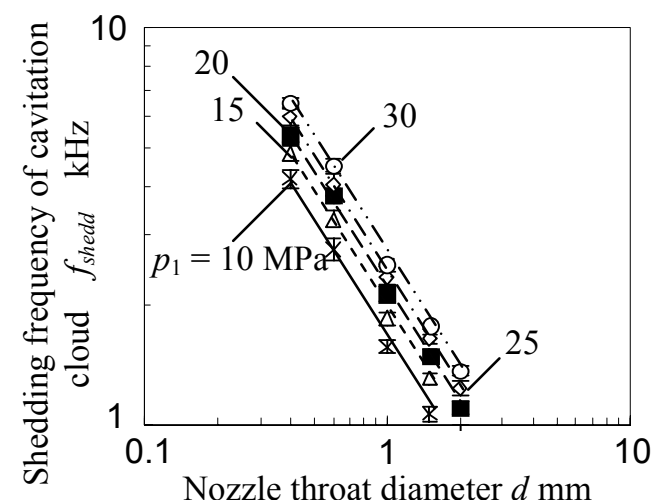

(a) $\sigma=0.014$

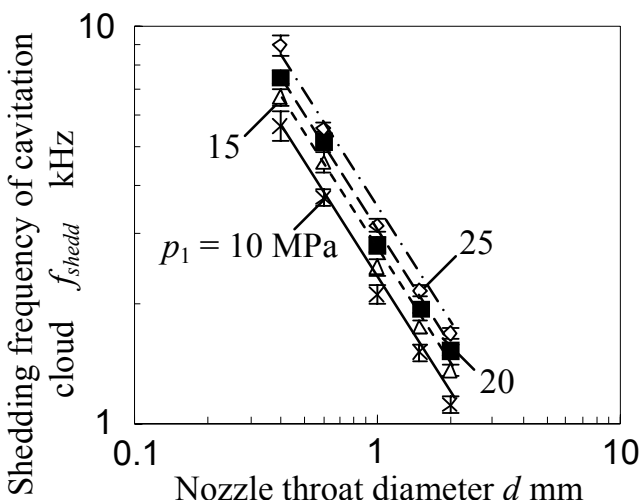

(b) $\sigma=0.02$

Fig. 9 Variation of the shedding frequency of a cavitation cloud with nozzle throat diameter at constant injection pressure 
relationship between $f_{\text {shedd }}$ and $d$ was obtained using the least squares method on the data in Figs. 8 and 9, and is given by Eq. (10)

$$
f_{\text {shedd }}=f_{c} d^{m}
$$

where $f_{c}$ and $m$ are a constant and a power index, respectively, which depend on the conditions. The standard deviations of $f_{c}$ and $m$ were calculated, and these are considered to be the experimental errors. The values of $f_{c}$ and $m$ for various injection pressure, $p_{1}$, are shown in Tables 2 and 3. It can be seen that $m$ is almost constant, with a value of about -1 , even though $p_{1}$ varies by a factor of 3 . As $m=-1$ is included within the error range for most of the conditions, it can be concluded that the relationship between $f_{\text {shedd }}$ and $d$ can be described by Eq. (7). The straight lines drawn in Figs. 8 and 9 using Eq. (7) fit well with the experimental data. On the other hand, $f_{c}$ increases with increasing $p_{1}$, as shown in Tables 2 and 3, and Fig. 10.

The constant, $f_{c}$, is plotted against injection pressure, $p_{1}$, in Fig. 10. The data is fitted to straight lines using the least squares method which gives the following relationship between $f_{c}$ and $p_{1}$;

$$
f_{c} \propto p_{1}^{0.45 \pm 0.03}
$$

It should be noted that the value of the power index is quite close to 0.5 , which suggests that there is good agreement between the experimental data and equation Eq. (5).

Table 2 Parameters of the experimentally derived equation depending on injection pressure $(\sigma=0.014)$

\begin{tabular}{ccc}
\hline \hline Injection pressure & Constant & Power index \\
\cline { 2 - 3 }$p_{1} \mathrm{MPa}$ & $f_{c} \mathrm{kHz}$ & $m$ \\
\hline 10 & $1.63 \pm 0.03$ & $-1.00 \pm 0.04$ \\
15 & $1.93 \pm 0.03$ & $-1.01 \pm 0.03$ \\
20 & $2.20 \pm 0.04$ & $-0.99 \pm 0.03$ \\
25 & $2.42 \pm 0.02$ & $-0.98 \pm 0.02$ \\
30 & $2.65 \pm 0.04$ & $-0.98 \pm 0.03$ \\
\hline \hline
\end{tabular}

Table 3 Parameters of the experimentally derived equation depending on injection pressure $(\sigma=0.02)$

\begin{tabular}{ccc}
\hline \hline Injection pressure & Constant & Power index \\
\cline { 2 - 3 }$p_{1} \mathrm{MPa}$ & $f_{c} \mathrm{kHz}$ & $m$ \\
\hline 10 & $2.20 \pm 0.03$ & $-1.01 \pm 0.04$ \\
15 & $2.61 \pm 0.02$ & $-1.01 \pm 0.02$ \\
20 & $2.97 \pm 0.02$ & $-1.00 \pm 0.03$ \\
25 & $3.33 \pm 0.04$ & $-1.04 \pm 0.04$ \\
\hline \hline
\end{tabular}

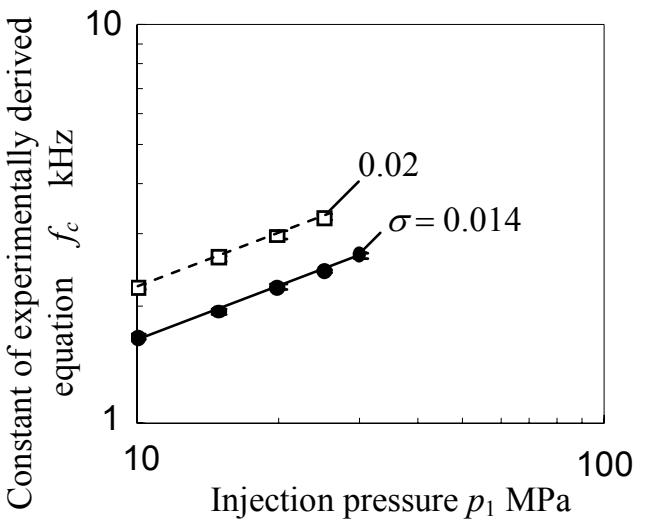

Fig. 10 Variation of $f_{c}$ with injection pressure 
Figure 11 shows the variation of shedding frequency, $f_{\text {shedd }}$, with injection pressure, $p_{1}$, for different values of $d$, whereas Fig. 12 shows the variation $f_{\text {shedd }}$ with $p_{1}$ for different values of $\sigma$. The common tendency shared among the figures and conditions is that $f_{\text {shedd }}$ increases with $p_{1}$, as expected from Eq. (5). The following equation for $f_{\text {shedd }}$ and $p_{1}$ was obtained using the least squares method;

$$
f_{\text {shedd }}=f_{c}{ }^{\prime} p_{1}{ }^{n}
$$

where, $f_{c}$, and $n$ are a constant and the power index, respectively, of the experimentally derived equation. Tables 4 and 5 show the values of $f_{c}$, and $n$, together with their standard deviations, for various nozzle throat diameter, $d$, and the two different values of cavitation number. It can be observed that the power index $n$ is almost constant at around 0.5 even
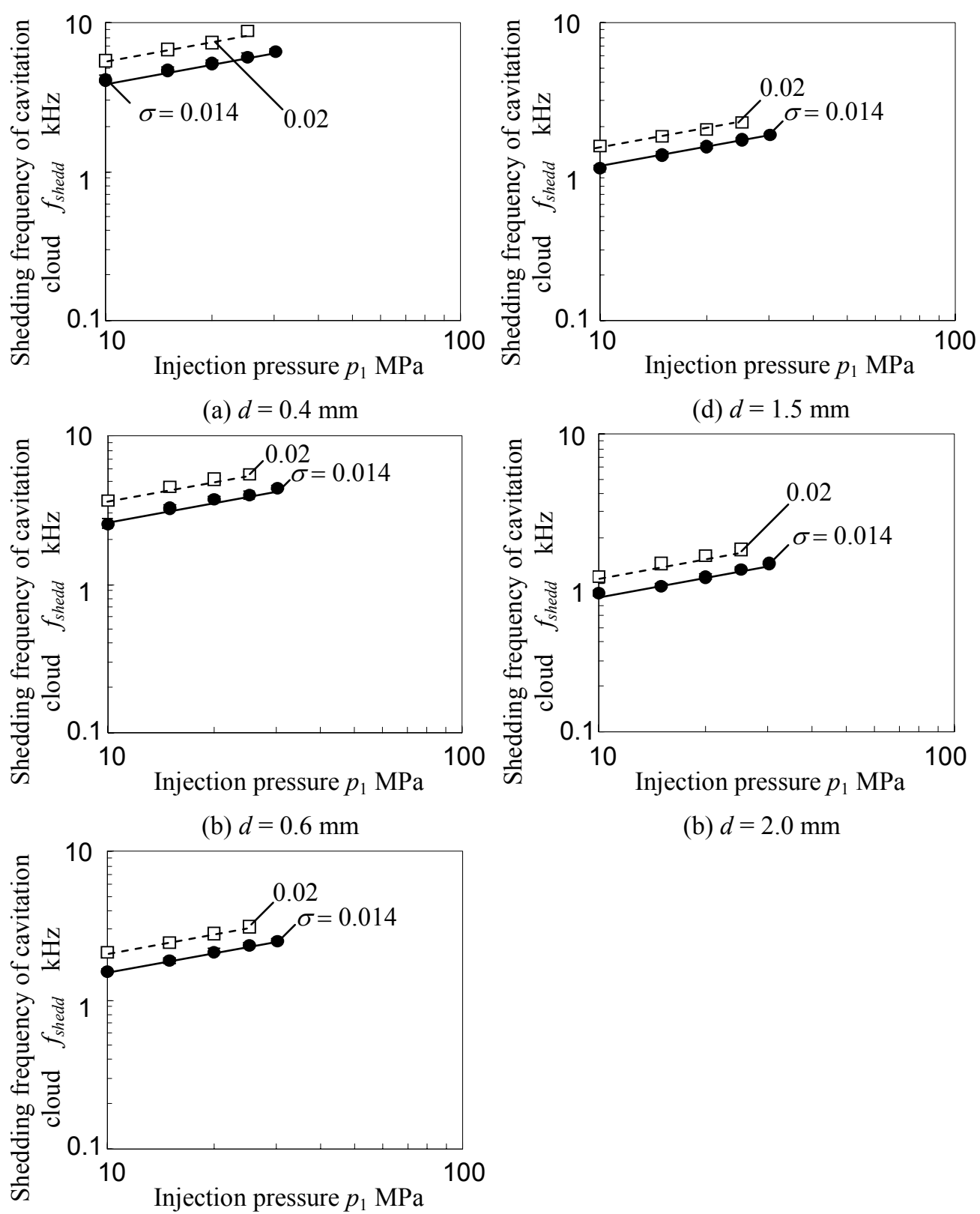

(b) $d=2.0 \mathrm{~mm}$

(c) $d=1.0 \mathrm{~mm}$

Fig. 11 Variation of the shedding frequency of a cavitation cloud with injection pressure at constant cavitation number 
though $d$ varies by a factor of 5 . Considering that $n=0.5$ is mostly within the error ranges in Tables 4 and 5, Eq. (5) can be considered to be a valid relationship for $f_{\text {shedd }}$ and $p_{1}$. The straight lines in Figs. 11 and 12 represent Eq. (5), which fit well with the experimental data. However, $f_{c}$ ' decreases with increasing $d$, as shown in Tables 4 and 5.

Figure 13 shows the constant in the experimental relationship given in Eq. (12), $f_{c}$, varying with the nozzle throat diameter, $d$. Using the least squares method, the following relationship between $f_{c}$, and $d$ is found;

$$
f_{c}^{\prime} \propto d^{0.98 \pm 0.14}
$$

This is in agreement with both Eqs. (7) and (10).

Figure 14 shows the shedding frequency, $f_{\text {shedd }}$, varying with cavitation number, $\sigma$, for different values of injection pressure, whereas Fig. 15 shows $f_{\text {shedd }}$ varying with $\sigma$ for different values of nozzle throat diameter. From the figures and conditions, a common trend

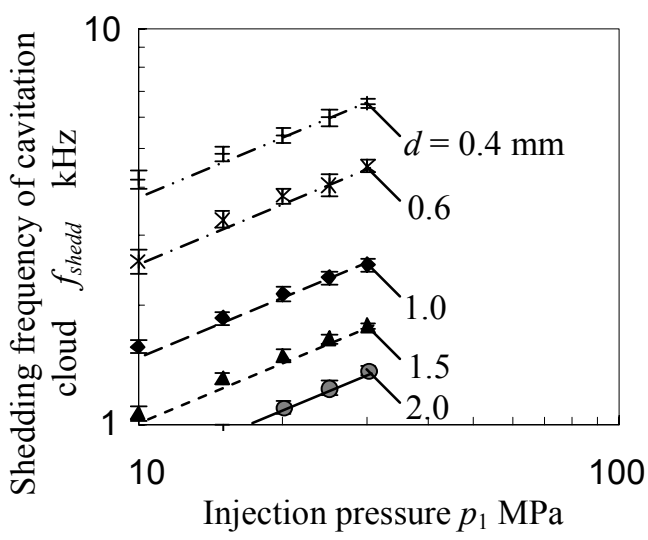

(a) $\sigma=0.014$

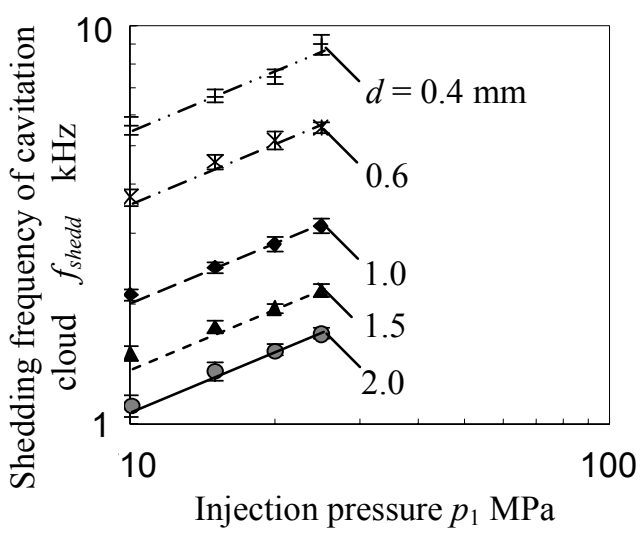

(b) $\sigma=0.02$

Fig. 12 Variation of the shedding frequency of a cavitation cloud with injection pressure at constant nozzle throat diameter

Table 4 Parameters of the experimentally derived equation depending on nozzle throat diameter $(\sigma=0.014)$

\begin{tabular}{ccc}
\hline \hline $\begin{array}{c}\text { Nozzle throat } \\
\text { diameter } d \mathrm{~mm}\end{array}$ & Constant & Power index \\
\cline { 2 - 3 }$f_{c} \mathrm{kHz}$ & $n$ \\
\hline 0.4 & $1.49 \pm 0.37$ & $0.43 \pm 0.07$ \\
0.6 & $0.88 \pm 0.17$ & $0.48 \pm 0.06$ \\
1.0 & $0.57 \pm 0.08$ & $0.44 \pm 0.05$ \\
1.5 & $0.38 \pm 0.04$ & $0.45 \pm 0.03$ \\
2.0 & $0.29 \pm 0.04$ & $0.45 \pm 0.04$ \\
\hline \hline
\end{tabular}

Table 5 Parameters of the experimentally derived equation depending on nozzle throat diameter $(\sigma=0.02)$

\begin{tabular}{ccc}
\hline \hline $\begin{array}{c}\text { Nozzle throat } \\
\text { diameter } d \mathrm{~mm}\end{array}$ & Constant & Power index \\
\cline { 2 - 3 } & $f_{c} \mathrm{kHz}$ & $n$ \\
\hline 0.4 & $1.83 \pm 0.42$ & $0.48 \pm 0.07$ \\
0.6 & $1.40 \pm 0.26$ & $0.43 \pm 0.06$ \\
1.0 & $0.78 \pm 0.12$ & $0.43 \pm 0.05$ \\
1.5 & $0.52 \pm 0.10$ & $0.44 \pm 0.06$ \\
2.0 & $0.40 \pm 0.09$ & $0.45 \pm 0.07$ \\
\hline \hline
\end{tabular}




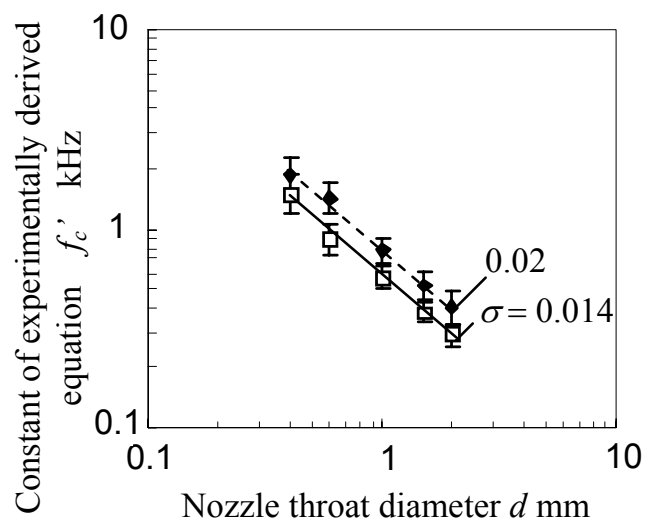

Fig. 13 Variation of $f_{c}$, with nozzle throat diameter

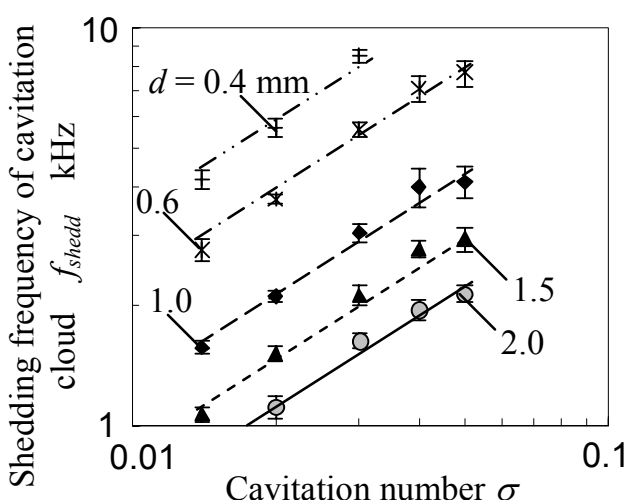

(a) $p_{1}=10 \mathrm{MPa}$

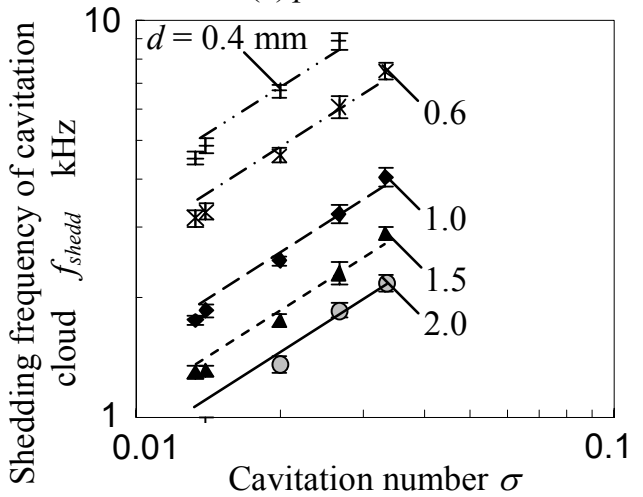

(b) $p_{1}=15 \mathrm{MPa}$

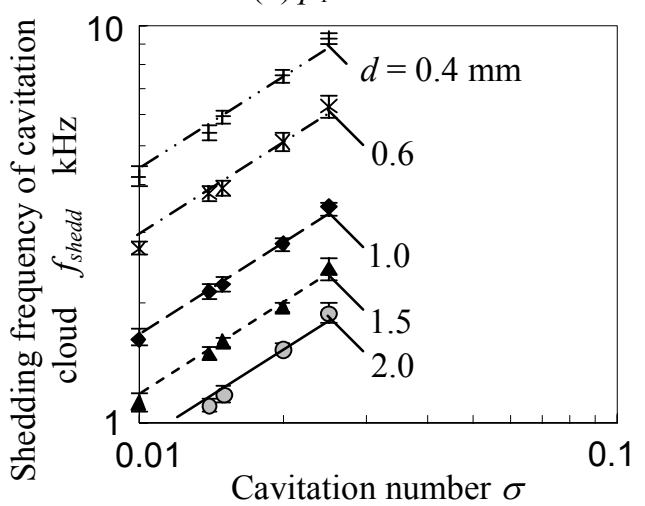

(c) $p_{1}=20 \mathrm{MPa}$

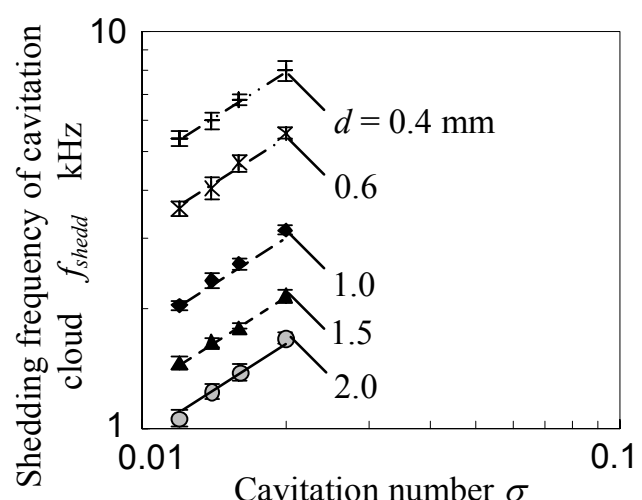

(d) $p_{1}=25 \mathrm{MPa}$

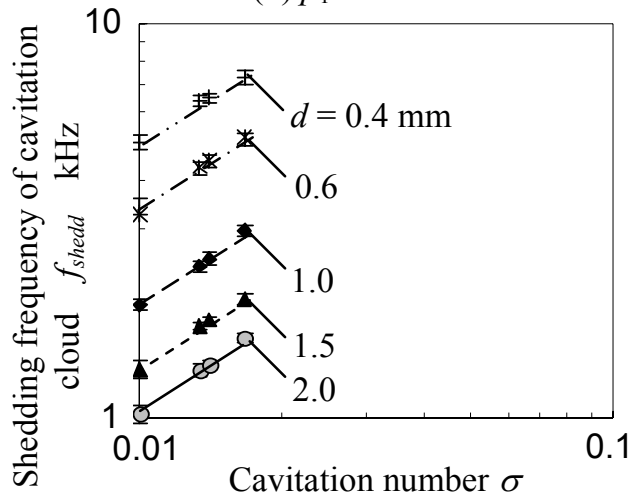

(e) $p_{1}=30 \mathrm{MPa}$

Fig. 14 Variation of the shedding frequency of a cavitation cloud with cavitation number at constant nozzle throat diameter 


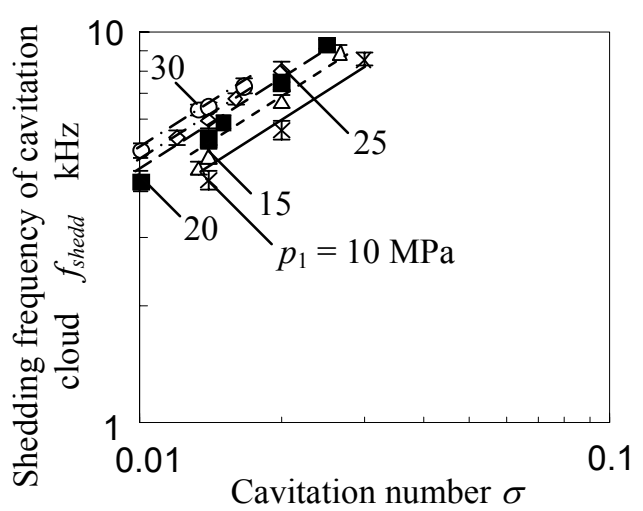

(a) $d=0.4 \mathrm{~mm}$

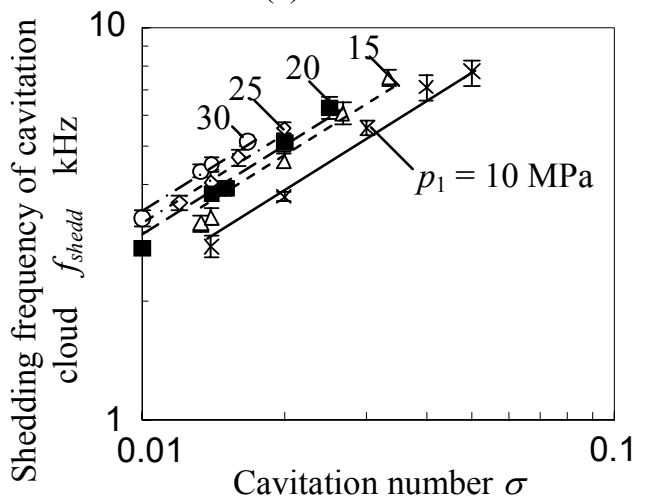

(b) $d=0.6 \mathrm{~mm}$

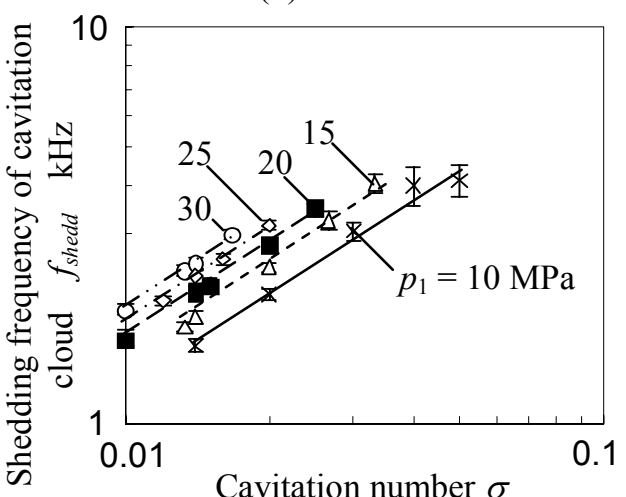

(c) $d=1.0 \mathrm{~mm}$

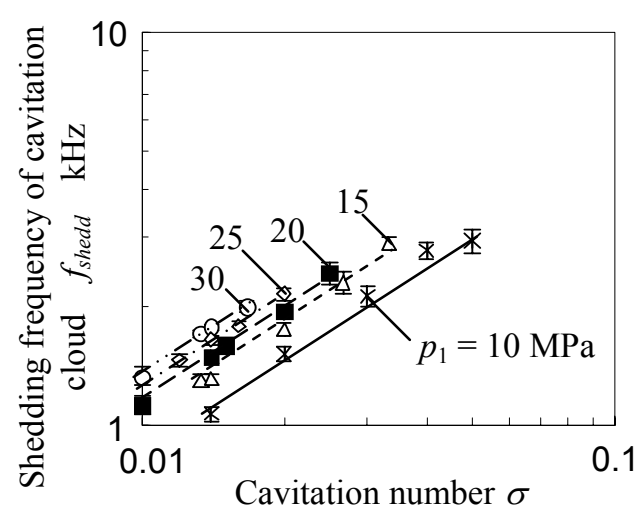

(d) $d=1.5 \mathrm{~mm}$

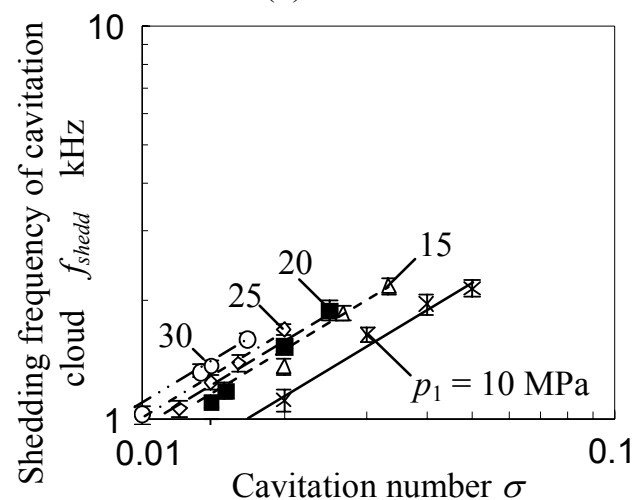

(d) $d=2.0 \mathrm{~mm}$

Fig. 15 Variation of the shedding frequency of a cavitation cloud with cavitation number at constant injection pressure

is revealed, i.e., $f_{\text {shedd }}$ increases with $\sigma$, as expected from Eq (9). Using the least squares method the experimental data in Figs. 14 and 15 can be fitted by the following equation;

$$
f_{\text {shedd }} \propto \sigma^{0.83 \pm 0.10}
$$

Here, it should be noted that the power index, $0.76 \pm 0.02$, in Eq. (9) is within the experimental error range of the power index, $0.83 \pm 0.10$, in Eq. (14), showing that there is good agreement between the two equations. Therefore, it can be concluded that the relationship between $f_{\text {shedd }}$ and $\sigma$ described by Eq. (9) has been validated by the experimental data in Figs. 14 and 15.

Figure 16 shows the relationship between the Strouhal number, St, of cavitation cloud shedding and the cavitation number, $\sigma$, calculated from the data of Figs. (6), (14) and (15). This shows $S t$ to be almost constant and independent of $\sigma$. Moreover, $S t$ is seen to be almost independent of the nozzle throat diameter, $d$, and the injection pressure, $p_{1}$, since this is different for $\sigma=0.014$ and 0.02 . The average value of $S t$ is $0.18 \pm 0.02$, where the error 
range was obtained from the standard deviation. As the value of $S t$ for cavitation cloud shedding is related to the vortex structure around the jet, the result of Fig. 16 demonstrates that the vortex structure around a cavitating jet does not depend on $d, p_{1}$ or $\sigma$, as long as similar shaped nozzles are used. This tendency agrees entirely with the similarity law for an unsteady vortex flow ${ }^{(18)}$.

The experimental results of this study affirm our assumption that cavitation cloud shedding induced by a cavitating jet follows the similarity law for unsteady vortex flow, and that this phenomenon is governed by a constant Strouhal number. The constant value of the Strouhal number should be unique to the nozzle outlet geometry, which affects the shedding frequency of cavitation cloud ${ }^{(15)}$. The Strouhal number for the nozzle outlet geometry kept in similar shape as $d: D: L=1: 8: 8$ is constant with a value of 0.18 , as presented here.

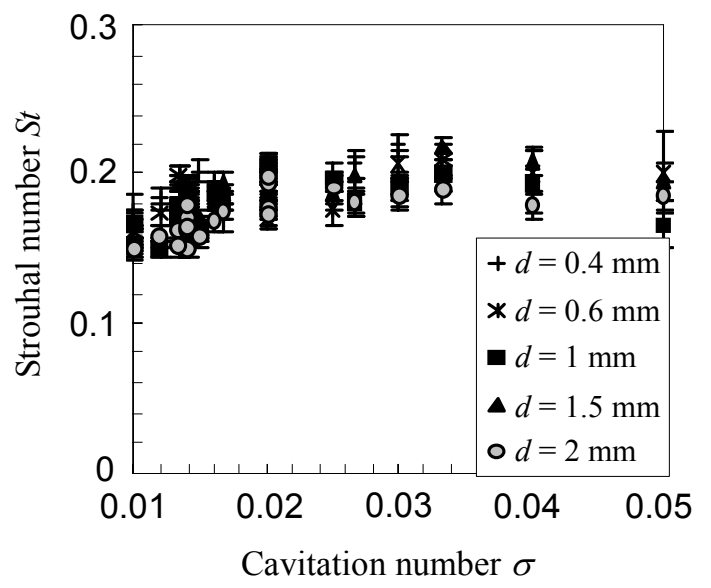

Fig. 16 Strouhal number for cavitation cloud shedding varying with cavitation number

\section{Conclusions}

Consideration of the practical applications of a cavitating jet directed us towards further studies of the mechanism of cavitation cloud shedding induced by a cavitating jet. In order to clarify the cavitation cloud shedding mechanism, the shedding frequencies of cavitation clouds produced with various nozzle throat diameters, injection pressures and cavitation numbers were investigated by high-speed observations and image analysis. In this paper, the nozzles were cylindrical, with geometrically similar dimensions, since the shedding frequency of the cavitation cloud depends on this.

The results presented here show that cavitation cloud shedding induced by a cavitating jet adhere to a similarity law. Cavitation cloud shedding is a phenomenon governed by a constant Strouhal number, St, which is defined in terms of the shedding frequency of the cavitation cloud, $f_{\text {shedd }}$, the width of the cavitating jet, $w$, and the velocity of the jet at nozzle exit, $U$, which depends on the injection pressure. The Strouhal number for the results presented here is constant with a value of 0.18 . Thus, for a fixed injection pressure, the frequency is inversely proportional to the width of the jet. Since the width of the jet is proportional to the nozzle throat diameter, $d$, the frequency is also inversely proportional to $d$. In addition, the shedding frequency increases in proportion with the square root of the injection pressure.

$$
\begin{aligned}
& S t=\frac{f_{\text {shedd }} \cdot w}{U} \cong 0.18 \pm 0.02 \\
& f_{\text {shedd }} \propto d^{-1} \\
& w \propto d
\end{aligned}
$$




$$
\begin{aligned}
& f_{\text {shedd }} \propto w^{-1} \\
& f_{\text {shedd }} \propto p_{1}^{0.5}
\end{aligned}
$$

\section{Acknowledgment}

This work was partly supported by the Japan Society for the Promotion of Science under the Grant-in-Aid for Scientific Research (B)24360040. The authors thank Mr. M. Mikami, technician, Tohoku University for his help in the experiment.

\section{Appendix}

In order to demonstrate the effect that the position of the analytical point has on the shedding frequency of the cavitation cloud, Fig. A1 shows the shedding frequency as a function of the distance from the nozzle exit, $x$, with $d=1 \mathrm{~mm}, p_{1}=30 \mathrm{MPa}$, and $\sigma=0.014$. The shedding frequency of the cavitation cloud, fshedd, is almost constant from $x=10 \mathrm{~mm}$ to $60 \mathrm{~mm}$, then decreases with increasing $x$. It can be assumed that the decrease in $f_{\text {shedd }}$ is due to the collapse of the cavitation cloud, considering that the optimum standoff distance is $53 \mathrm{~mm}^{(15)}$.

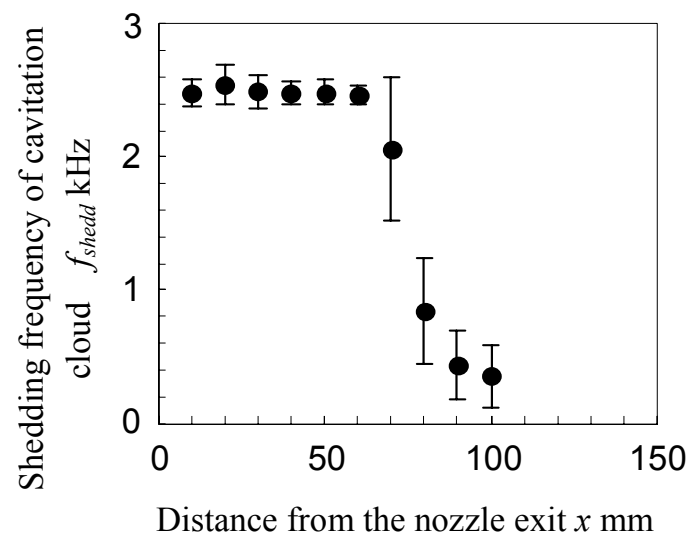

Fig. A1 Influence of the analysis point on the shedding frequency of the cavitation cloud

\section{Nomenclature}

$d=$ Nozzle throat diameter

$\sigma=$ Cavitation number

$p_{1}=$ Injection pressure

$p_{2}=$ Ambient pressure

$p_{v}=$ Vapor pressure

$D=$ Nozzle outlet bore

$L=$ Nozzle outlet length

$x=$ Distance from the nozzle exit

$f_{\text {shedd }}=$ Shedding frequency of the cavitation cloud

$w=$ Width of the cavitating jet

$U=$ Velocity of the jet at the nozzle exit

$S t=$ Strouhal number

$\rho=$ Density of the water

$f_{c}=$ Constant of the experimentally derived equation

$f_{c}{ }^{\prime}=$ Constant of the experimentally derived equation 
$m=$ Power index

$n=$ Power index

\section{References}

1. Soyama, H. and Sekine, Y., Sustainable Surface Modification Using Cavitation Impact for Enhancing Fatigue Strength Demonstrated by a Power Circulating-Type Gear Tester, International Journal of Sustainable Engineering, Vol. 3, No. 1 (2010), pp. 25-32.

2. Johnson, Jr. V.E., Lindenmuth, W.T., Conn, A.F. and Frederick, G.S., Feasibility Study of Tuned-Resonator, Pulsating Cavitating Water Jet for Deep-Hole Drilling, Sandia National Laboratories, Contractor Report SAND81-7126, (1981), pp. 1-131.

3. Kalumuck, K.M. and Chahine, G.L., The Use of Cavitating Jets to Oxidize Organic Compounds in Water, Transactions of the ASME, Journal of Fluids Engineering, Vol. 122, No. 3 (2000), pp. 465-470.

4. Ooi, K.K., Scale Effects on Cavitation Inception in Submerged Water Jets : a New Look, Journal of Fluid Mechanics, Vol. 151 (1985), pp. 367-390.

5. Ran, B. and Katz, J., Pressure Fluctuations and their Effect on Cavitation Inception within Water Jets, Journal of Fluid Mechanics, Vol. 262 (1994), pp. 223-263.

6. Gopalan, S., Katz, J. and Knio, O., The Flow Structure in the Near Field of Jets and its Effect on Cavitation Inception, Journal of Fluid Mechanics, Vol. 398 (1999), pp. 1-43.

7. Straka, A.S., Meyer, S.R., Fontaine, A.A. and Welz, J.P., Cavitation Inception in Quiescent and Co-Flow Nozzle Jets, Journal of Hydrodynamics, Series B, Vol. 22, No. 5, Supplement 1 (2010), pp. 813-819.

8. Vijay, M.M., Zou, C. and Tavoularis, S., A Study of the Characteristics of Cavitating Water Jets by Photography and Erosion, Proceedings of the 10th International Conference on Jet Cutting Technology, (1991), pp. 37-67.

9. Chahine, G.L., Johnson, Jr., V.E., Kalumuck, K.M., Perdue, T.O., Waxman, D.N., Frederick, G.S. and Watson, R.E., Internal and External Acoustics and Large Structure Dynamics of Cavitating Self-Resonating Water Jets, Sandia National Laboratories, Contractor Report SAND86-7176, (1987), pp. 1-202.

10. Soyama, H., Yamauchi, Y., Adachi, Y., Sato, K., Shindo, T. and Oba, R., High-Speed Observations of the Cavitation Cloud around a High-speed Submerged Water Jet, JSME International Journal, Series B, Vol. 38, No. 2 (1995), pp. 245-251.

11. Hulti, E.A.F. and Nedeljkovic, M.S., Frequency in Shedding/ Discharging Cavitation Clouds Determined by Visualization of a Submerged Cavitating Jet, Transactions of the ASME, Journal of Fluids Engineering, Vol. 130 (2008), pp.021304-1-8.

12. Sato, K., Sugimoto, Y. and Ohjimi, S., Structure of Periodic Cavitation Clouds in Submerged Impinging Water-Jet Issued form Horn-Type Nozzle, Proceedings of the 9th Pacific Rim International Conference on Water Jetting Technology, (2009).

13. ASTM Designation G134-95, Standard Test Method for Erosion of Solid Materials by a Cavitating Liquid Jet, Annual Book of ASTM Standards, Vol. 3, No. 2 (2006), pp. 559-571.

14. Brennen, C.E., Cavitation and Bubble Dynamics, (1995), Oxford University Press.

15. Soyama, H., Enhancing the Aggressive Intensity of a Cavitating Jet by Means of the Nozzle Outlet Geometry, Transactions of the ASME, Journal of Fluids Engineering, Vol. 133, No. 3 (2011), pp. 101301-1-11.

16. Soyama, H., Material Testing and Surface Modification by Using Cavitating Jet, Journal of the Society of Materials Science, Vol. 47, No. 8 (1998), pp. 381-387.

17. Soyama, H. and Lichtarowicz, A., Cavitating Jets - Similarity Correlations, Journal of Jet Flow Engineering, Vol. 13, No. 2 (1996), pp. 9-19.

18. Bearman, P.W., On Vortex Street Wakes, Journal of Fluid Mechanics, Vol. 28 (1967), pp. 625-641. 